\title{
Upper extremity deep vein thrombosis: pathogenesis and treatment
}

\author{
Piotr Pukacki, Robert Juszkat, Maciej Błaszyk, Łukasz Dzieciuchowicz, Zbigniew Krasiński \\ University Hospital of Lord's Transfiguration, partner of Karol Marcinkowski University of Medical Sciences in Poznań, Poland
}

\begin{abstract}
Upper extremity deep vein thrombosis (UEDVT) may be the first manifestation of venous thoracic outlet syndrome (VTOS). It primarily affects young, physically active people. The clinical findings depend on the degree of obstruction of the subclavian vein. Correct diagnosis — aided by various imaging modalities — as well as rapid initiation of local thrombolytic therapy, surgical decompression of the thoracic outlet (when indications are present), and the immediate initiation of anticoagulation therapy aim at successfully restoring the patient's quality of life.
\end{abstract}

Key words: upper extremity deep vein thrombosis, thoracic outlet syndrome, subclavian vein thrombosis, paget-schroetter syndrome, pathogenesis and treatment

Acta Angiol 2019; 25, 2: I15-119

\section{Introduction}

It is estimated that venous thromboembolic diseases occur in approximately 200-300 people per 100000 persons annually. One-third of these patients presents with a pulmonary embolism (PE), while two-thirds present with deep vein thrombosis (DVT). Upper extremity deep vein thrombosis (UEDVT) accounts for approximately $4-10 \%$ of all cases of DVT [1, 2]. Depending on the etiology, upper extremity DVT can be classified as either primary or secondary [3-6].

Primary UEDVT includes spontaneous thromboembolic events "without clear cause," that may involve an underlying latent thrombophilia, or the not yet fully understood phenomenon of effort thrombosis, also known as Paget-Schroetter syndrome [7].

Secondary UEDVT refers to thrombosis in the setting of endothelial trauma caused by indwelling devices (most commonly central venous catheters, pacemaker electrodes and leads) and malignancy [3].

The relationship between upper extremity deep vein thrombosis and physical effort (effort thrombosis) was first observed in 1875 by Paget and then by Von Schroetter inspiring them to search for the cause of this complication. Currently it is believed that subclavian vein thrombosis develops as a consequence of repetitive microtrauma due to the compression present in thoracic outlet syndrome (TOS) [1, 8]. This term was first introduced by Peet in 1956 [9]. It encompasses the constellation of clinical findings that may arise as a consequence of compression of the neurovascular structures passing through the narrow, limited costoclavicular space from the chest towards the upper extremity $[9,10]$. The subclavian vein, a continuation of the axillary vein, and as one of these structures, traverses the costoclavicular space with the subclavius muscle. It runs anteriorly to the anterior scalene muscle, enters the supraclavicular space, where posteriorly to the manubrium it joins the internal jugular vein. This space is limited anteriorly, from the manubrium, by the costoclavicular ligament. Extreme positioning of the limb or physical effort changes the anatomical conditions, which causes venous microtrauma with endothelial damage and precipitates thrombosis [I I].

Address for correspondence: Piotr Pukacki, University Hospital of Lord's Transfiguration, partner of Karol Marcinkowski University of Medical Sciences in Poznań, Poland, Długa I/2, 6I-848 Poznań, Poland, e-mail: piotr.pukacki@gmail.com 
It may lead to a venous compression syndrome which some authors refer to as "nutcracker syndrome" [12]. This explains the occurrence of acute DVT in young, physically active people without a clear cause.

Primary upper extremity deep vein thrombosis may be associated with physical effort in as high as $20-30 \%$ of cases and is a type of venous thoracic outlet syndrome (VTOS) [I]. Many researchers consider this percentage to be overstated, however, in view of the complex pathophysiology and the favorable long-term outcomes of proper treatment, VTOS is still a current topic of discussion [13].

Clinical diagnosis of subclavian vein thrombosis due to compression may prove difficult, especially when the thrombus involves only a short segment of the vessel. In this case, the clinical manifestations are non-specific, which yields an error rate of approx. $50 \%$ and results in erroneous or delayed treatment [10]. The classical symptoms of early (10-14 days) upper extremity deep vein thrombosis are a consequence of venous congestion. Swelling of the upper limb is the dominating symptom, along with changes in skin color, pain during active movements or when pressure is applied to the muscles. Late symptoms include the dilation of collateral veins in the arm and upper part of the chest. The patient's medical history from the period preceding the onset of thrombosis is underestimated. Often, patients report discomfort while performing everyday housework involving lifting of the upper limbs. However, provocative tests for signs of compression and assessments of blood flow are unreliable. Though a positive test result may suggest venous compression, objective imaging studies indicate that $40 \%$ of the healthy population has a positive test result with no clinical signs of compression $[14,15]$. This opinion is shared by Moore, who believes that the classic Adson's maneuver is not applicable in current VTOS diagnosis [12].

For patients for whom the diagnosis of UEDVT is "highly probable," we perform additional radiologic imaging examinations such as Doppler ultrasound, chest $\mathrm{X}$-ray, computed tomography angiography or magnetic resonance angiography. These methods allow us to accurately determine the anatomic conditions as well as the hemodynamic significance of compression and the degree of venous obstruction [3].

An initial plain radiograph of the thoracic outlet widely available, inexpensive and safe — is performed to rule out any underlying bone abnormalities that may be the cause of the analyzed compression symptoms and various forms of TOS - cervical ribs, bony anomalies of the first rib, clavicle, enlarged transverse processes of the last cervical vertebrae, or scarring after clavicle fracture. However, it should be remembered that this is only an initial imaging study.
Ultrasonography is the first-line imaging modality in the diagnosis of UEDVT [10, 16]. It is performed after an initial clinical examination of the patient. For the diagnosis of DVT, its sensitivity is estimated at $78-100 \%$ and specificity at $82-100 \%$ [12]. More importantly, veins may be examined dynamically in various positions of the upper limb, which increases the imaging method's diagnostic value [14, 16]. Changing the position of the limb makes it easier to determine whether the vein is compressed from the outside, and also visualize the location of stenosis within the vein. Duplex ultrasonography can also be used to assess blood flow. Significant flow acceleration indicates narrowing and compression, while an absence of flow suggests complete occlusion of the vessel. However, the accuracy of the examination depends on the anatomical conditions of the patient and the examiner's experience.

According to one group of researchers, duplex ultrasonography does not definitively exclude upper extremity deep vein thrombosis [17]. Bony structures are one obstacle in the assessment [16]. Another aspect that makes it difficult to assess subclavian vein thrombosis is the development of good collateral circulation with rapid blood flow. In view of the above-mentioned limitations, in order to definitively assess thrombotic changes in the veins of the upper extremity, contrast-enhanced imaging modalities are used. This is particularly important in the assessment of chronic venous thrombosis lasting more than 6 weeks.

According to Vemuri, CT angiography is a valuable supplement to non-invasive ultrasonography in locating a compression of the proximal segment of the subclavian vein. Additionally, axial scans provide information on the soft tissue structures (tumor, lymph nodes, thrombus, vessel wall) [I, 3].

Angio-MR is another recommended imaging modality in the assessment of venous compression syndromes due to fibromuscular tissues. However, this examination requires more time in order to obtain the final result $[3,16]$.

Venography is the oldest and most invasive diagnostic method since it involves a catheter being introduced into the vein and the injection of contrast. It is used when the diagnosis of compression is uncertain and for planned venous reconstructive surgery [I]. It remains the "golden standard" in the assessment of venous pathology and plays an important role intraoperatively in the assessment of blood flow through the subclavian vein both prior to and following reconstructive or decompression surgery. It determines the extent and location of obstruction, the state of the collateral circulation, and the changes in blood flow in the vessel lumen [18]. 
Complications of UEDVT include pulmonary embolism (PE) and post-thrombotic syndrome (PTS). PE is diagnosed in $2-5 \%$ of patients with UEDVT, and according to some authors, even reaches up to $20 \%$ [I]. However, this type of complication is more often observed in secondary DVT $[7,8]$. There are also authors who believe that the percentage of emboli originating in the deep veins of the upper limbs is unknown, because patients who do not present obvious symptoms of venous stasis are not examined for thrombosis [1, 19]. Therefore, an ultrasound examination of the veins of the upper limbs in search of the origin of pulmonary emboli should be performed each time the cause is unknown [I]. Apart from $\mathrm{PE}$, other clinically relevant complications of DVT include: persistent or increased swelling of the limb after physical activity, bruising of the skin, heaviness of the affected limb, dilation of skin vessels or early recurrence of DVT. The above symptoms are referred to as late complications or post-thrombotic syndrome - PTS. It is estimated that it occurs in 7 to $46 \%$ of cases of DVT. PTS is inseparably connected with the underlying cause of UEDVT and its ineffective treatment, which usually only involves anticoagulation therapy [I]. As is the case in May-Thurner syndrome, conservative treatment does not ensure complete restoration of patency $[2,20]$. Therefore, after a period of improvement, the recurrence of DVT is observed, along with aggravated symptoms of impaired venous return, which leads to a proportion of patients with PTS and functional impairment of the affected limb.

The following elements should be considered in the management of UEDVT: rapid restoration of adequate blood flow with thromboprophylaxis, suppression of thrombus formation and inflammatory processes and restoration of limb function [2I].

Secondary DVT, characterized by the presence of diagnostic catheters, pacemakers or malignancy in the vicinity of the vessel, is treated in accordance with the established guidelines based on randomized clinical trials and clinical practice. It is not recommended to remove all "foreign bodies", especially when they are an important element in the treatment of the underlying disease. The mainstay of conservative treatment is anticoagulant therapy - low molecular weight heparin (LMWH) in therapeutic doses for 5-7 days with the exception of hemodynamically unstable patients. Patients do not require laboratory monitoring beyond a periodic evaluation of platelet count. Monitoring anti-Xa activity is limited to cases of individual LMWH dosing in patients with renal insufficiency and extreme obesity. LMWH is used in conjunction with Vitamin $\mathrm{K}$ antagonists (VKA) until a stable INR value is achieved in the therapeutic range between 2-3. The acute phase of secondary DVT can also be treated with an intravenous infusion of unfractionated heparin UFH, monitoring its effectiveness by the elongation of the aPTT ratio by $1.5-2$ times control in a repeat assay, 6 hours after its initiation. A new group of anticoagulants that has proven convenient and valuable in the treatment of DVT are direct thrombin inhibitors (factor Ila), such as Dabigatran, and direct factor $\mathrm{Xa}$ inhibitors (Rivaroxaban, Apixaban, Endoxaban). These Non-VKA Oral Anticoagulants (NOAC) selectively inhibit a specific coagulation factor [18]. When diagnosing primary, effort thrombosis in the course of VTOS in a young patient, in addition to restoring the patency of the subclavian vein, we strive to eliminate the root cause of thrombotic complications. We are treating a "healthy" patient who is professionally active and expects the full restoration of limb function. Based on the analysis of the available literature and the procedures adopted at an institution located in Poznan, it seems that the most appropriate course of action to achieve the above objective is the initial administration of LMWH with the concomitant introduction of a catheter into the subclavian vein and the performance of venography. Upon confirmation of thrombosis, local catheter-directed thrombolysis (CDT) is initiated. Two main types of thrombolytic drugs are currently in use: urokinase and rtPA. In the regimen adopted by the Department of Vascular and Intravascular Surgery, Angiology and Phlebology at Poznan University of Medical Sciences, rtPA is administered directly to the thrombus at a rate of $\mathrm{I} \mathrm{mg} / \mathrm{h}$ with constant monitoring of fibrinogen, aPTT and platelet count. The maximum duration of treatment is 72 hours. Although in most cases acute (up to 14 days) DVT undergoes local thrombolysis, in some patients residual thrombi may remain, which pose a risk of recurrence of thrombosis [10]. With the aim of restoring vein patency, sometimes more aggressive treatment is proposed in the form of a percutaneous pharmacomechanical thrombectomy (PMT) [12]. This method combines the local administration of a thrombolytic with the mechanical removal of the thrombus. Depending on the mechanism of thrombus removal, the following types of mechanical thrombectomy are distinguished: rotational (e.g. Aspirex), aspiration (e.g. Indigo system), rheolytic (e.g. Angiojet) and ultrasound-enhanced. The reduced thrombus burden after this type of treatment gives the opportunity to restore blood flow already after the first procedure [17]. Monitoring of the patient requires increased postoperative vigilance due to the risk of developing hemolytic complications.

A review of the literature and data from various centers dealing selectively with VTOS shows that the mainstay of UEDVT treatment, in addition to the immediate restoration of blood flow, is the surgical decompression of the subclavian vein by first rib resection 
along with the adjacent fibromuscular tissues [I I, 20, $22,23]$. The timing of the surgery and the extent of the procedure remains the topic of discussion. Advocates of urgent first rib resection explain their rationale with a concern for the recurrence of thrombosis [24]. Another option is performing the surgical decompression within 4-6 weeks following fibrinolytic treatment [24-26]. In both cases, an extremely important component of further management and prognosis following the restoration of subclavian vein patency is its radiological assessment.

Algorithms for dealing with UEDVT have been introduced $[25,26]$. The assumption of the adopted VTOS treatment was the rapid restoration of the original venous blood flow (the "open vein" concept) and the surgical removal of extravascular and intravascular changes within the subclavian vein. Although the final, long-term outcomes were improved, a percentage of patients still remained with a functional impairment of the limb. In 2017, the work of Vazquez et al. was published, which analyzed 25 studies, in which the results of treatment of 127 I patients with UEDVT were discussed [13]. The resection of the first rib was obligatory for all patients. The primary criterion of assessment was the development of PTS, while the secondary criterion was: DVT recurrence, $\mathrm{PE}$, death, hemorrhage as well as re-operation with or without an endovascular procedure. In the final conclusions, the authors found that anticoagulant therapy alone does not protect the patient from the development of PTS and that surgical treatment with or without fibrinolysis is necessary to improve the final outcomes, which translates into an improved quality of life for the patients [ I, I3]. Therefore, modern surgical treatment of VTOS involves the complete decompression of the subclavian vein and ensuring optimal blood flow. Vemuri et al. [I] recommend surgery after $4-6$ weeks of effective thrombolysis. This prevents the recurrence of thrombosis and allows for the resolution of the adjacent inflammatory processes, while early (immediate) decompression is recommended in patients with severe obstruction. Moreover, they believe that the prophylactic decompression of incidentally detected venous compression is unnecessary [12].

There are three main surgical approaches to subclavian vein decompression. The first: transaxillary first rib resection with partial scalenectomy. This method was described and widely used by Machleder since 1990 [II, 25, 26]. The procedure requires extensive knowledge of anatomy and surgical skills. The advantage of the method is a small, cosmetic postoperative scar and the creation of a wide space (following first rib resection) for the passing neurovascular structures. The disadvantage of this method is a small operative field and the inability to directly reconstruct the damaged vein.
The next step in the procedure is an evaluation of the subclavian vein in venography after 2-3 weeks and in the case of persistent stenosis, balloon venoplasty with the continuation of oral anticoagulant therapy. The use of stents in the subclavian vein is not recommended, particularly in cases where first rib resection is not performed [23].

The infraclavicular approach described by Molina et al. [20] provides a good intraoperative view. It allows for wide exposure of the subclavian vein following the excision of the subclavius muscle, the costoclavicular ligament and the resection of the anterior part of the first rib. Such access facilitates the reconstruction of the vessel with the use of the patient's great saphenous vein, insertion of a venous patch widening the vessel lumen or a biological patch. According to the authors of the method, the procedure should not be carried out more than two weeks after the onset of thrombotic symptoms or in patients with a long-segment occlusion or a venous diameter of less than $1 \mathrm{~cm}$. The reconstruction surgery should be followed after 24 hours with a venogram [20]. Both methods give similar outcomes: $75-80 \%$ of patients with VTOS remain without symptoms with a patent subclavian vein. Only in $20-25 \%$ of cases complications of thrombosis are observed, which are an indication for anticoagulant therapy [I].

The third group of proponents of aggressive decompression of the subclavian vein in the thoracic outlet is composed of surgeons performing the resection from both the supraclavicular and infraclavicular approach. Good access to the regional anatomy obtained in this approach increases the extent and radicality of the resected bone and fibromuscular structures compressing the vein. The aim of the surgery is the total resection of the first rib as well as the reconstruction of the scarred vein under visual inspection. Most often, these authors perform a reconstruction of the affected vein with an autologous venous patch or a bovine pericardial patch. In the case of vessel obstruction, the occluded fragment of the subclavian vein is replaced by a cryopreserved femoral vein $[2,5,27]$. In the case of a venous graft, a peripheral arteriovenous fistula is necessary [27]. The surgical procedure is supplemented by venography or intravascular ultrasound (IVUS), which provides objective information about the patency of the "decompressed" vein $[2,28]$. Oral anticoagulant therapy is recommended for a minimum of three months, and 6-12 weeks after surgery patients can resume all normal activities. In addition to the advantages of aggressive UEDVT treatment, the possible complications should be mentioned. The less serious complications include: pneumothorax, hematoma, lymphedema or post-operative wound infection. In view of the infrequency of the performed operations the following 
severe complications are described in the literature: permanent damage to the brachial plexus, damage to the long thoracic nerve, diaphragmatic nerve or finally recurrent thrombosis of the reconstructed subclavian vein [I]. The possibility of developing late complications of UEDVT, the need for precise diagnostic imaging, and the identification of an effective treatment method all point to the need to create centers specializing in the treatment of upper limb thrombosis.

\section{Conflict of interest}

None.

\section{References:}

I. Vemuri C, Salehi P, Benarroch-Gampel J, et al. Diagnosis and treatment of effort-induced thrombosis of the axillary subclavian vein due to venous thoracic outlet syndrome. J Vasc Surg Venous Lymphat Disord. 2016; 4(4): 485-500, doi: 10.1016/j. jvsv.2016.01.004, indexed in Pubmed: 27639006.

2. Lee JA, Zierler BK, Zierler RE. The risk factors and clinical outcomes of upper extremity deep vein thrombosis. Vasc Endovascular Surg. 2012; 46(2): 139-144, doi: |0.1 I77/I5385744 | |432 |45, indexed in Pubmed: 22328450.

3. "Imaging in Deep Venous Thrombosis of the Upper Extremity: Practice Essentials, Radiography, Computed Tomography," Sep. 2018.

4. Heil J, Miesbach W, Vogl T, et al. Deep Vein Thrombosis of the Upper Extremity. Dtsch Arztebl Int. 2017; II4(14): 244-249, doi: 10.3238/arztebl.2017.0244, indexed in Pubmed: 2844635 I.

5. Carlon TA, Sudheendra D. Interventional Therapy for Upper Extremity Deep Vein Thrombosis. Semin Intervent Radiol. 2017; 34(I): 54-60, doi: 10.1055/s-0036-1597764, indexed in Pubmed: 28265130 .

6. Klitfod L, Broholm R, Baekgaard N. Deep venous thrombosis of the upper extremity. A review. Int Angiol. 2013; 32(5): 447-452, indexed in Pubmed: 2390330I.

7. Illig KA, Doyle AJ. A comprehensive review of Paget-Schroetter syndrome. J Vasc Surg. 20 10; 5 I (6): 1538-1547, doi: 10.1016/j. jvs.2009.12.022, indexed in Pubmed: 20304578.

8. Thompson RW. Comprehensive management of subclavian vein effort thrombosis. Semin Intervent Radiol. 2012; 29(I): 44-5I, doi: 10. 1055/s-0032-I30245I, indexed in Pubmed: 23448848.

9. Peet RM, Henriksen JD, Anderson TP, et al. Thoracic-outlet syndrome: evaluation of a therapeutic exercise program. Proc. Staff Meet. Mayo Clin. 1956; 31(9): 28I-287, doi: 10.1007/ springerreference_3985I.

10. Archie M, Rigberg D. Vascular TOS-Creating a Protocol and Sticking to It. Diagnostics (Basel). 2017; 7(2), doi: 10.3390/diagnostics7020034, indexed in Pubmed: 28604581.

II. de León RA, Chang DC, Hassoun HT, et al. Multiple treatment algorithms for successful outcomes in venous thoracic outlet syndrome. Surgery. 2009; 145(5): 500-507, doi: 10.1016/j. surg.2008.09.017, indexed in Pubmed: 19375608.

12. Moore R, Wei Lum Y. Venous thoracic outlet syndrome. Vasc Med. 2015; 20(2): 182-189, doi: 10.1 I77//358863X14568704, indexed in Pubmed: 25832605.
13. Vazquez FJ, Paulin P, Poodts D, et al. Preferred management of primary deep arm vein thrombosis. Eur J Vasc Endovasc Surg. 2017; 53(5): 744-75I, doi: 10.1016/j.ejvs.2016.1 I.028, indexed in Pubmed: 2834273।.

14. Urbanek T, Ziaja D, Kwiecień A, et al. Występowanie zespołu uciskowego górnego otworu klatki piersiowej (TOS) w populacji w wieku 19-26 lat. Chir Pol . 2008 ; 10(I): 8-15.

15. Chen $\mathrm{H}$, Doornbos $\mathrm{N}$, Williams K, et al. Physiologic variations in venous and arterial hemodynamics in response to postural changes at the thoracic outlet in normal volunteers. Ann Vasc Surg. 2014; 28(7): 1583-1588, doi: 10.1016/j.avsg.2014.05.003, indexed in Pubmed: 24909503.

16. Zucker EJ, Ganguli S, Ghoshhajra BB, et al. Imaging of venous compression syndromes. Cardiovasc Diagn Ther. 2016; 6(6): 519-532, doi: 10.21037/cdt.2016.11.19, indexed in Pubmed: 28123973.

17. Kärkkäinen JM. Pharmacomechanical thrombectomy in Paget-Schroetter syndrome. Cardiovasc Intervent Radiol Sep. 2016; 39(9): 1272-1279.

18. Kearon C, Akl EA, Ornelas J, et al. Antithrombotic therapy for VTE disease: CHEST Guideline and Expert Panel Report. Chest. 2016; 149(2): 315-352, doi: 10.1016/j.chest.2015.11.026, indexed in Pubmed: 26867832.

19. Hobeika C, Meziane A, Sands MJ, et al. Paget-Schroetter syndrome: an uncommon cause of pulmonary embolic disease. J Thorac Imaging. 2010; 25(I): I-3.

20. Molina JE, Hunter DW, Dietz CA. Paget-Schroetter syndrome treated with thrombolytics and immediate surgery. J Vasc Surg. 2007; 45(2): 328-334, doi: 10.1016/j.jvs.2006.09.052, indexed in Pubmed: 17264012.

2I. Siracuse JJ, Johnston PC, Jones DW, et al. Infraclavicular first rib resection for the treatment of acute venous thoracic outlet syndrome. J Vasc Surg Venous Lymphat Disord. 2015; 3(4): 397-400, doi: 10.1016/j.jvsv.2015.06.002, indexed in Pubmed: 26992617.

22. Hussain MA, Aljabri B, Al-Omran M. Vascular Thoracic Outlet Syndrome. Semin Thorac Cardiovasc Surg. 2016; 28(I): 15I157, doi: 10.1053/j.semtcvs.2015.10.008, indexed in Pubmed: 27568153.

23. Freischlag J. Venous thoracic outlet syndrome: transaxillary approach. Operative Techniques in General Surgery. 2008; 10(3): 122-130, doi: 10.1053/j.optechgensurg.2008.09.003.

24. Urschel HC, Patel AN. Surgery remains the most effective treatment for Paget-Schroetter syndrome: 50 years' experience. Ann Thorac Surg. 2008; 86(I): 254-60; discussion 260, doi: 10.1016/j. athoracsur.2008.03.02I, indexed in Pubmed: 18573433.

25. Machleder $\mathrm{H}$. The anterior scalene muscle in thoracic outlet compression syndrome. Archives of Surgery. 1986; 121(10): II4I, doi: 10.1001/archsurg.1986.01400100047009.

26. Chang KZ, Likes K, Demos J, et al. Routine venography following transaxillary first rib resection and scalenectomy (FRRS) for chronic subclavian vein thrombosis ensures excellent outcomes and vein patency. Vasc Endovascular Surg. 2012; 46(I): 15-20, doi: 10.1 I77/I 5385744 I I 423982, indexed in Pubmed: 22156I50.

27. Rodriguez HE, Yao ST, Pearce WH. The ischemic extremity: new findings and treatment. PMPH-USA. 2010.

28. Molina JE, Hunter DW, Dietz CA. Protocols for Paget-Schroetter syndrome and late treatment of chronic subclavian vein obstruction. Ann Thorac Surg. 2009; 87(2): 416-422, doi: 10.1016/j. athoracsur.2008.1 I.056, indexed in Pubmed: 19161749. 